Proceedings

\title{
Chemopreventive Doses of Curcumin Protect Cells from Cadmium Induced Oxidative Stress ${ }^{\dagger}$
}

\author{
Maria Russo *, Stefania Moccia, Carmen Cervellera, Carmela Spagnuolo, Idolo Tedesco, \\ Paola Minasi, Virginia Carbone, Maria Grazia Volpe and Gian Luigi Russo \\ Published: date \\ Academic Editor: name
}

National Research Council, Institute of Food Sciences, 83100 Avellino, Italy; stefania.moccia@isa.cnr.it (S.M.); carmencervellera@libero.it (C.C.); carmela.spagnuolo@isa.cnr.it (C.S.); idolo.tedesco@isa.cnr.it (I.T.); paola.minasi@isa.cnr.it (P.M.); virginia.carbone@isa.cnr.it (V.C.); mgvolpe@isa.cnr.it (M.G.V.); glrusso@isa.cnr.it (G.L.R.)

* Correspondence: maria.russo@isa.cnr.it; Tel.: +39-0825-299331

+ Presented at the 1st International e-Conference on Antioxidants in Health and Disease, 1-15 December 2020; Available online: https://cahd2020.sciforum.net/.

Published: 30 November 2020

\begin{abstract}
Circumstantial evidence link the high levels of environmental pollutants to increased incidence of different chronic-degenerative diseases. Among these, the levels of heavy metals (HM) can certainly play a key role. On the other hand, a qualified literature highlights how different phytochemicals, normally present in the diet are able to interfere with HM metabolism helping to reduce tissue concentrations and their negative effects on health. Curcumin (Cur) appears of particular interest for its protective properties against cadmium (Cd) toxicity. HL-60 cells, differentiated in human monocyte/macrophage lineage by vitamin D analogue EB1089, were preincubated with low doses of Cur $(1 \mu \mathrm{M})$ and treated for $24 \mathrm{~h}$ with $15 \mu \mathrm{M}$ Cd. Cell viability was measured using CyQuant fluorescent dye. ROS (reactive oxygen species) and reduced glutathione (GSH) variations were assayed respectively by dichlorofluorescein-diacetate method and monochlorobimane probe. The bioavailability of Cur in HL-60 cells was successfully performed using RP-HPLC coupled with UV-Vis, MS/MS or MS/MSn. In differentiated HL-60 cells, the preincubation with $1 \mu \mathrm{M}$ Cur determined a significant $20 \%$ protection towards the cytotoxicity induced by $\mathrm{Cd}$. The investigation of the mechanism of action evidenced that curcumin lowered ROS increase caused by $\mathrm{Cd}$ by increasing GSH intracellular levels. The antioxidant activity of Cur was strengthened by the detection of free curcumin inside the cells after 5 min of treatment.
\end{abstract}

Keywords: curcumin; cadmium; intracellular ROS; environmental pollutants; dietary chemoprevention

\section{Introduction}

Heavy metals, (HM) unlike organic contaminants, are not biodegradable and comprise elements like cadmium $(\mathrm{Cd})$, lead $(\mathrm{Pb})$, arsenic $(\mathrm{As})$, mercury $(\mathrm{Hg})$, and chromium $(\mathrm{Cr})$, which are located along the Earth's crust in various forms. Environmental pollution of soil, water, and air by HM is a global problem due to anthropogenic activities, ranging from agriculture to industry [1]. In recent years has become increasingly evident the role of these environmental pollutants in influencing 
The 1st International Electronic Conference on Antioxidants in Health and Disease, 1-15 December 2020

human health: European Environmental Agency have calculated about 500,000 premature deaths and diseases caused by indoor and outdoor air pollution in the European Union [2]. An analysis of the countries with more deaths attributed to air pollution reveals that Italy, Germany, Poland, France and UK, in this order, are at the top of the list. In Italy, there is an area called "the Land of Fire" located between Naples and Caserta in Campania region, that has become the symbol of a national ecological crisis whose echo has gone beyond national borders, contributing to damage the overall image of an entire region and negatively affect, above all, the agri-food sector [3]. Scientific literature reported several studies related to the impact of HM pollution in this area on human health [4,5]. In particular, different studies highlighted a worrying increase in HM at concentrations high enough to affect the health of animals [6] and humans in terms of acute and chronic toxicity (alteration of related parameters reproduction, endocrine system, immunosuppression) [7]. Qualified literature highlights how different phytochemical compounds, normally present in the diet, are able to interact with HM, interfering with their cellular metabolism and helping to reduce tissue concentrations [8]. Curcumin (Cur) is a polyphenol (1,7-bis (4-hydroxy-3-methoxyphenyl) -1,6-heptadiene-3,5-dione) that different studies have indicated as the main active compound of turmeric against chronic diseases including various types of cancer, diabetes, obesity, cardiovascular, pulmonary, neurological and autoimmune diseases $[9,10]$. Different studies associate nutritional interventions with protection from health damage caused by HM: the combination of Cur $(50 \mathrm{mg} / \mathrm{kg})$ and Resveratrol $(20 \mathrm{mg} / \mathrm{kg}) \mathrm{reduced}$ oxidative damage caused by $\mathrm{Cd}$ in a mouse model of liver toxicity [11]. A different study, conducted in rat model, concluded that Cur $(30 \mathrm{mg} / \mathrm{kg})$ can exert a neuroprotective effect by chelating HM such as $\mathrm{Pb}$ and $\mathrm{Cd}$ [12]. The formation of Cur-HM complexes would reduce oxidative stress (increase in reactive oxygen species, ROS) at the cellular and tissue level [10]. To effectively translate animal studies in human's clinical trials based on nutritional supplements with phytochemicals, it is well known that a major problem is their rapid metabolism and low bioavailability after oral ingestion [13]. Primary aim of this preclinical study is the use of very low doses of Cur $(1 \mu \mathrm{M})$ to verify the mechanism of Cur protection against Cd toxicity. We used an in vitro model of human myelomonocytic cells obtained after in vitro differentiation of HL-60 myelocitic blasts with vitamin D analogue EB1089 [14]. Results obtained demonstrate for the first time a protective role of low doses of Cur against $\mathrm{Cd}$ toxicity due to a rapid intracellular uptake and efficient antioxidant response. These findings could be useful to formulate a Cur enriched "functional" food and/or nutraceutical supplement to be tested in a clinical trial finalized to protect population living in HM contaminated area.

\section{Materials and Methods}

\subsection{Cell Culture and Viability Assay}

HL-60 cells (EACC) [15] were cultured in RPMI medium supplemented with 10\% FBS, 1\% Lglutamine and $1 \%$ penicillin/streptomycin (Euroclone, Milan, Italy) at $37{ }^{\circ} \mathrm{C}$ in a humidified atmosphere containing 5\% $\mathrm{CO}_{2}$. Cell differentiation was obtained after one week supplementation with 100 nM vitamin D (VD) analogue EB1082 (Sigma/Aldrich, Milan) [14]. Differentiated HL-60 cells were preincbated overnight. with $1 \mu \mathrm{M}$ Cur (Sigma/Aldrich) or $0.1 \%$ DMSO (CTRL). Day after, medium was changed and cells were incubated with $15 \mu \mathrm{M} \mathrm{Cd}$ Nitrate $\left(\mathrm{Cd}\left(\mathrm{NO}_{3}\right)_{2}\right.$, Sigma/Aldrich) for additional $24 \mathrm{~h}$. Cellular viability was assessed with CyQuant dye (Invitrogen, TermoFischer, Milan, Italy) as described [16]. After cell staining, microphotographs were captured by fluorescence inverted microscope (Axiovert Zeiss) at 400× magnification using FITC filter.

\subsection{Intracellular ROS}

Intracellular ROS were evaluated with CM-DCFDA intracellular fluorescent probe (TermoFischer-Scientific) after overnight Cur $1 \mu \mathrm{M}$ pretreatment. Cells were washed and incubated for 5 min with complete RPMI medium or adding $15 \mu \mathrm{M} \mathrm{Cd}$ [17]. 
The 1st International Electronic Conference on Antioxidants in Health and Disease, 1-15 December 2020

\subsection{GSH Assay}

Reduced glutathione (GSH) intracellular content was measured after 6 h of incubation with 15 $\mu \mathrm{M}$ Cd after overnight pretreatment with $1 \mu \mathrm{M}$ Cur, by using Monochlorobimane as fluorescent probe (MCB, Sigma/Aldrich) [18]. $\mathrm{H}_{2} \mathrm{O}_{2} 100 \mu \mathrm{M}$ was used as GSH depleting agent.

\subsection{Measurement of Intracellular Levels of Cur in HL-60}

To detect intracellular levels of Cur, HL-60 cells $\left(1.5 \times 10^{6}\right)$ were incubated with $1 \mu \mathrm{M}$ Cur in complete RPMI cell culture medium. After 5 min cells were washed with PBS, resuspended 3 times in methanol and sonicated. Cur concentration in cell extracts was evaluated as described, using RPHPLC coupled with UV-Vis, MS/MS or MS/MSn [16].

\subsection{Statistical Analysis}

The data were obtained from at least two separate experiments and presented as mean \pm standard deviation (SD). The Student's $t$-test in Microsoft Excel 2019 program was used to analyze the statistical significance between different treatments and control groups.

\section{Results}

\subsection{Low Dose of Curcumin Rescues Cell Viability Due to Cd Toxicity in Differentiated HL-60 Cells after Overnight Incubation}

To evaluate protective effects of Cur in a cell model resembling human macrophage/monocyte cells, HL-60 were differentiated $168 \mathrm{~h}$ with vitamin D analogue EB1089 (100 nM). Differentiated HL60 cells were viable but not proliferating. They were incubated with $1 \mu \mathrm{M}$ Cur overnigh in complete medium. Cur concentration was not toxic in these conditions because we calculated an EC-50 of 20 $\mu \mathrm{M}$. Day after, medium was changed and replaced with Cd $15 \mu \mathrm{M}$ for additional $24 \mathrm{~h}$. Results described in Figure 1a, after CyQuant dye fluorescence quantification, show that Cd is very toxic in these conditions $(<45 \%$ cell viability, EC-50 $=25 \mu \mathrm{M})$. However, Cur preincubation could significatively rescue ( $>20 \%, p<0.001$ vs. Cd) cell viability in HL-60 differentiated cells. These results are visualized in micrographs in Figure $1 \mathrm{~b}-\mathrm{e}$, showing representative fields in the indicated experimental conditions after incubation with CyQuant fluorescent dye.

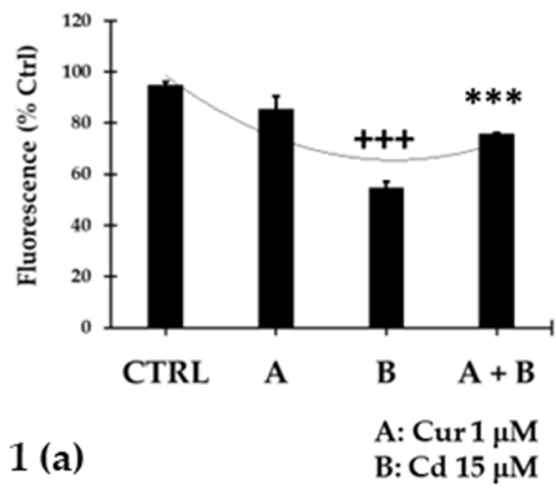

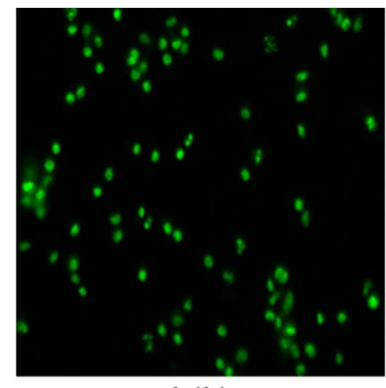

1 (b)

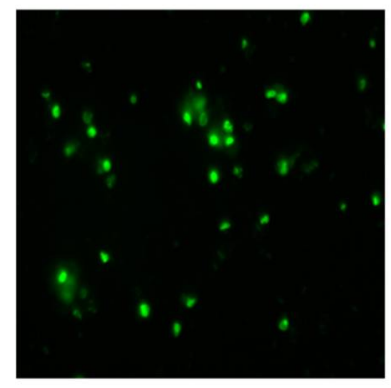

1(d)

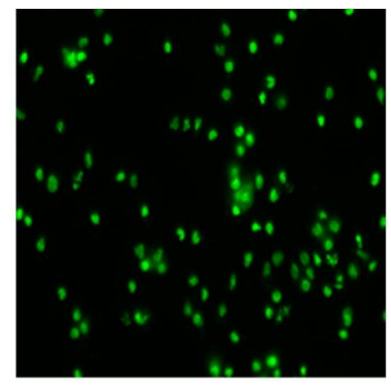

1(c)

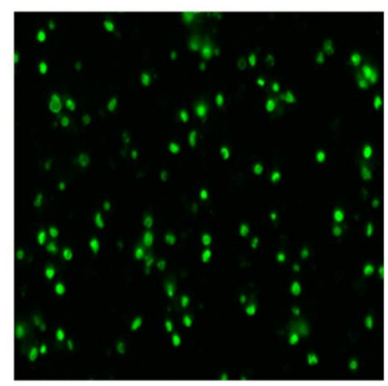

1(e) 
Figure 1. Cur protects differentiated HL-60 cells from Cd toxicity. (a) CyQuant viability assay after 1 $\mu \mathrm{M}$ Cur overnight pre-incubation and replacement with medium or Cd $15 \mu \mathrm{M}$ for additional $24 \mathrm{~h}$. Results are expressed as percentage of CyQuant dye fluorescence respect to $0.1 \%$ DMSO treated cells (CTRL). Micrographs show representative fields of different experimental points: (b) CTRL, (c) Cur, (d) Cd, (e) Cd after pre-incubation with Cur (400×, FITC filter). Symbols indicate statistical significance after $t$-test Student: $+++p<0.001$ vs. CTRL, ${ }^{* * *} p<0.001$ vs. Cd.

\subsection{Curcumin Pretreatment Lowers Intracellular ROS Generated by $\mathrm{Cd}$}

The main factor affecting Cd toxicity and carcinogenicity is production of ROS [10]. With the generation of peroxides, non-fluorescent CM-DCFH-DA dye is oxidized to fluorescent CM-DCF that, in turn, is quantitatively measured with a fluorescence reader. Figure $2 a$ shows that $15 \mu \mathrm{M} \mathrm{Cd}$, soon after addition in cell culture medium $(5 \mathrm{~min})$, significally rised intracellular peroxides respect to CTRL $(>10 \% p<0.001)$ and Cur pre-treated cells $(>15 \% p<0.001)$. Overnight incubation with Cur slightly lowered intracellular ROS respect to CTRL $(<5 \% p<0.05)$. When HL-60 cells, pre-treated with Cur, were incubated with $\mathrm{Cd}$, the percentage of cells displaying ROS generation was significantly decreased probably due to Cur pre-treatment $(<15 \% p<0.001$ vs. Cd).

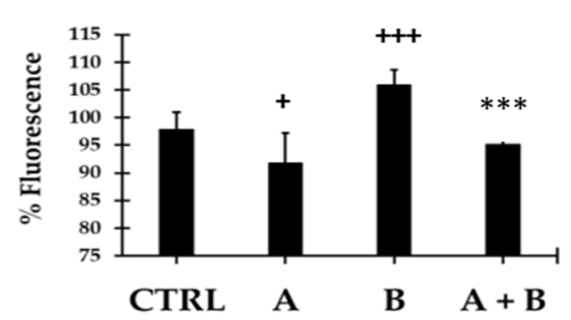

2 (a)

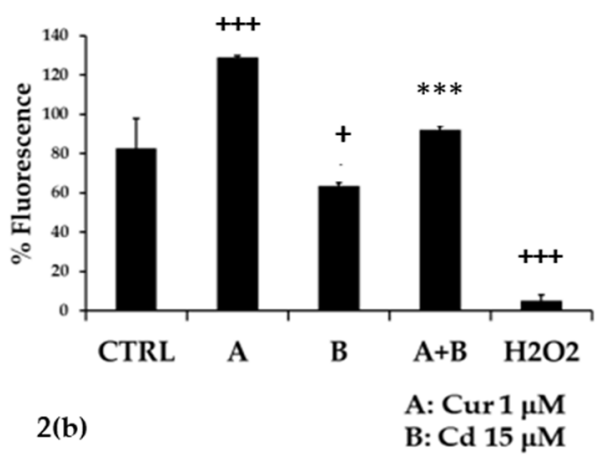

Figure 2. Cur protect HL-60 cells from ROS and GSH depletion induced by Cd. (a) Intracellular ROS measurement obtained with CM-DCFDA probe after $1 \mu \mathrm{M}$ Cur (A) overnight pre-incubation and replacement with medium or Cd $15 \mu \mathrm{M}$ (B) for $5 \mathrm{~min}$. Results are expressed as percent of DCF fluorescence respect to $0.1 \%$ DMSO treated cells (CTRL). (b) GSH intracellular content measured with fluorescent probe $\mathrm{MCB}$, after overnight Cur incubation (A) and replacement for $6 \mathrm{~h}$ with medium, Cd $15 \mu \mathrm{M}(\mathrm{B})$ or $\mathrm{H}_{2} \mathrm{O}_{2} 200 \mu \mathrm{M}$. Symbols indicate statistical significance after $t$-test Student: $+p<0.05$ vs. CTRL, ${ }^{+++} p<0.001$ vs. CTRL, ${ }^{* * *} p<0.001$ vs. Cd.

\subsection{Curcumin Protect Cells from GSH Depletion Induced by Cd by Rising Its Intracellular Levels}

The toxicity of $\mathrm{Cd}$, as a redox-inactive metal, arises from the depletion of antioxidant GSH and bonding to the sulfhydryl groups in proteins [10]. Since a method for GSH assessment using bimanes could be rapid, sensitive and specific, we used monochlorobimane (MCB) as an intracellular fluorescent probe to verify if Cd induced toxicity in HL-60 was due to GSH depletion [18]. After $6 \mathrm{~h}$ of incubation with Cd, GSH levels were significantly lower than CRTL (Figure $2 b,<20 \% p<0.05$ ). On the opposite, Cur pre-treatment significantly rised intracellular GSH levels (Figure $2 b,>40 \% p<0.001$ vs. untretaed cells) as well as when $\mathrm{Cd}$ was added after Cur overnight pre-incubation (Figure $2 \mathrm{~b}$, $>30 \% p<0.001$ vs. Cd alone). $\mathrm{H}_{2} \mathrm{O}_{2}$ was used as GSH depleting agent.

\subsection{Curcumin Uptake in HL-60 Cells}

Due to the short half-life of Cur in aqueous medium, it is difficult to determine whether the observed biological actions of this polyphenol in vitro are due to Cur itself or its degradation products, hampering mechanistic understanding of Cur biology [19]. So, we developed a method to 
The 1st International Electronic Conference on Antioxidants in Health and Disease, 1-15 December 2020

extract Cur from HL-60 cells, soon after (5 min) addition in cell culture medium [16]. Table 1 shows that free Cur is present at measurable levels in cells after $5 \mathrm{~min}$ of incubation but, as aspected, it is rapidly degraded during time (starting from 1 until $15 \mathrm{~h}$ of incubation, not shown). This result revealed that Cur, al low levels, could be rapidly absorbed in HL-60 cells where it could exert antioxidant activities against $\mathrm{Cd}$ toxicity.

Table 1: Curcumin uptake in HL-60 cells

\begin{tabular}{ccc}
\hline CTRL DMSO & Treatment $(\mathrm{min})$ & Curcumin concentration \\
\hline N.D. & 5 & $1 \mu \mathrm{M}$ \\
\hline *numbers indicate ng curcumin $/ 1,5 \times 10^{6}$ cells \pm s.d. & \\
\hline
\end{tabular}

\section{Discussion}

This paper shows that low concentrations of Cur are able to protect human cells from Cd immunotoxicity. Since there are no papers in PubMed database using low doses of Cur in a cellular model of human myeloid lineage, this study is a novelty. Cur is rapidly absorbed in HL-60 but its intracellular concentration rapidly decline. In our conditions, Cur is eliminated from cell culture medium before adding $\mathrm{Cd}$, so it is not acting as a direct ROS scavenger in these experiments. However, Cur pre-treatment "prepare" cells to the oxidative insult induced by Cd by rising intracellular GSH content, probably by acting on Nuclear Factor Erythroid 2-Related Factor 2 (Nrf2) antioxidant system and activating an autophagic, protective process [20]. In these experimental conditions, low doses of Cur pre-treatment permit to evaluate "chemopreventive" efficacy of this molecule against $\mathrm{Cd}$ derived cellular damages. This experimental design could mimic the "realistic" intracellular concentrations of a dietary phytochemical present in cells of immunity system after a nutritional supplementation in humans [13].

Author Contributions: The following statements should be used M.R. conceived, designed the experiments and wrote the manuscript; M.R., S.M., C.S., C.C., I.T., V.C., P.M. performed the experiments and analyzed the data; M.G.V. and G.L.R. provided ideas and financial support. All authors have read and agreed to the published version of the manuscript.

Funding: This research received no external funding.

Acknowledgments: This work was financially supported by the program FESR 2014-2020 Campania Region "New formulations of functional nutraceutical products for the primary prevention of diseases associated with environmental pollutants in the Land of Fires" (EcoNutraPrevention). We kindly acknowledge all EcoNutraPrevention consortium.

Conflicts of Interest: The authors declare no conflict of interest.

\section{Abbreviations}

The following abbreviations are used in this manuscript:

$\begin{array}{ll}\text { HM } & \text { Heavy Metals } \\ \text { ROS } & \text { Reactive Oxygen Species } \\ \text { DMSO } & \text { Dimethyl sulfoxide } \\ \text { Cur } & \text { Curcumin } \\ \text { Cd } & \text { Cadmium Nitrate } \\ \text { GSH } & \text { reduced Glutathione } \\ \text { VD } & \text { Vitamin D } \\ \text { CM-DCFDA } & \text { 5-(and-6)-chloromethyl-2' }, 7^{\prime} \text {-dichlorodihydrofluorescein diacetate } \\ \text { MCB } & \text { Monochlorobimane }\end{array}$


The 1st International Electronic Conference on Antioxidants in Health and Disease, 1-15 December 2020

\section{References}

1. Rana, M.N.; Tangpong, J.; Rahman, M.M. Toxicodynamics of Lead, Cadmium, Mercury and Arsenicinduced kidney toxicity and treatment strategy: A mini review. Toxicol. Rep. 2018, 5, 704-713, doi:10.1016/j.toxrep.2018.05.012.

2. Carvalho, H. Air pollution-related deaths in Europe-Time for action. J. Glob. Health 2019, 9, 020308, doi:10.7189/jogh.09.020308.

3. Esposito, F.; Nardone, A.; Fasano, E.; Scognamiglio, G.; Esposito, D.; Agrelli, D.; Ottaiano, L.; Fagnano, M.; Adamo, P.; Beccaloni, E.; et al. A systematic risk characterization related to the dietary exposure of the population to potentially toxic elements through the ingestion of fruit and vegetables from a potentially contaminated area. A case study: The issue of the "Land of Fires" area in Campania region, Italy. Environ. Pollut. 2018, 243, 1781-1790, doi:10.1016/j.envpol.2018.09.058.

4. Mazza, A.; Piscitelli, P.; Falco, A.; Santoro, M.L.; Colangelo, M.; Imbriani, G.; Idolo, A.; De Donno, A.; Iannuzzi, L.; Colao, A. Heavy Environmental Pressure in Campania and Other Italian Regions: A Short Review of Available Evidence. Int. J. Environ. Res. Public Health 2018, 15, 105, doi:10.3390/ijerph15010105.

5. Triassi, M.; Alfano, R.; Illario, M.; Nardone, A.; Caporale, O.; Montuori, P. Environmental pollution from illegal waste disposal and health effects: A review on the "triangle of death". Int. J. Environ. Res. Public Health 2015, 12, 1216-1236, doi:10.3390/ijerph120201216.

6. Zaccaroni, A.; Corteggio, A.; Altamura, G.; Silvi, M.; Di Vaia, R.; Formigaro, C.; Borzacchiello, G. Elements levels in dogs from "triangle of death" and different areas of Campania region (Italy). Chemosphere 2014, 108, 62-69, doi:10.1016/j.chemosphere.2014.03.041.

7. Ebrahimi, M.; Khalili, N.; Razi, S.; Keshavarz-Fathi, M.; Khalili, N.; Rezaei, N. Effects of lead and cadmium on the immune system and cancer progression. J. Environ. Health Sci. Eng. 2020, 18, 335-343, doi:10.1007/s40201-020-00455-2.

8. Zhai, Q.; Narbad, A.; Chen, W. Dietary strategies for the treatment of cadmium and lead toxicity. Nutrients 2015, 7, 552-571, doi:10.3390/nu7010552.

9. Shanmugam, M.K.; Rane, G.; Kanchi, M.M.; Arfuso, F.; Chinnathambi, A.; Zayed, M.E.; Alharbi, S.A.; Tan, B.K.; Kumar, A.P.; Sethi, G. The multifaceted role of curcumin in cancer prevention and treatment. Molecules 2015, 20, 2728-2769, doi:10.3390/molecules20022728.

10. Mohajeri, M.; Rezaee, M.; Sahebkar, A. Cadmium-induced toxicity is rescued by curcumin: A review. BioFactors 2017, 43, 645-661, doi:10.1002/biof.1376.

11. Eybl, V.; Kotyzova, D.; Koutensky, J. Comparative study of natural antioxidants - Curcumin, resveratrol and melatonin-In cadmium-induced oxidative damage in mice. Toxicology 2006, 225, 150-156, doi:10.1016/j.tox.2006.05.011.

12. Daniel, S.; Limson, J.L.; Dairam, A.; Watkins, G.M.; Daya, S. Through metal binding, curcumin protects against lead- and cadmium-induced lipid peroxidation in rat brain homogenates and against lead-induced tissue damage in rat brain. J. Inorg. Biochem. 2004, 98, 266-275, doi:10.1016/j.jinorgbio.2003.10.014.

13. Russo, M.; Spagnuolo, C.; Tedesco, I.; Russo, G.L. Phytochemicals in cancer prevention and therapy: Truth or dare? Toxins 2010, 2, 517-551, doi:10.3390/toxins2040517.

14. James, S.Y.; Williams, M.A.; Kelsey, S.M.; Newland, A.C.; Colston, K.W. The role of vitamin D derivatives and retinoids in the differentiation of human leukaemia cells. Biochem. Pharmacol. 1997, 54, 625-634, doi:10.1016/s0006-2952(97)00195-0.

15. Breitman, T.R.; Selonick, S.E.; Collins, S.J. Induction of differentiation of the human promyelocytic leukemia cell line (HL-60) by retinoic acid. Proc. Natl. Acad. Sci. USA 1980, 77, 2936-2940, doi:10.1073/pnas.77.5.2936.

16. Russo, M.; Milito, A.; Spagnuolo, C.; Carbone, V.; Rosen, A.; Minasi, P.; Lauria, F.; Russo, G.L. CK2 and $\mathrm{PI} 3 \mathrm{~K}$ are direct molecular targets of quercetin in chronic lymphocytic leukaemia. Oncotarget 2017, 8, 4257142587, doi:10.18632/oncotarget.17246.

17. Wojtala, A.; Bonora, M.; Malinska, D.; Pinton, P.; Duszynski, J.; Wieckowski, M.R. Methods to monitor ROS production by fluorescence microscopy and fluorometry. Methods Enzymol. 2014, 542, 243-262, doi:10.1016/B978-0-12-416618-9.00013-3.

18. Capek, J.; Hauschke, M.; Bruckova, L.; Rousar, T. Comparison of glutathione levels measured using optimized monochlorobimane assay with those from ortho-phthalaldehyde assay in intact cells. J. Pharmacol. Toxicol. Methods 2017, 88, 40-45, doi:10.1016/j.vascn.2017.06.001. 
The 1st International Electronic Conference on Antioxidants in Health and Disease, 1-15 December 2020

19. Nimiya, Y.; Wang, W.; Du, Z.; Sukamtoh, E.; Zhu, J.; Decker, E.; Zhang, G. Redox modulation of curcumin stability: Redox active antioxidants increase chemical stability of curcumin. Mol. Nutr. Food Res. 2016, 60, 487-494, doi:10.1002/mnfr.201500681.

20. Guo, S.; Long, M.; Li, X.; Zhu, S.; Zhang, M.; Yang, Z. Curcumin activates autophagy and attenuates oxidative damage in EA.hy926 cells via the Akt/mTOR pathway. Mol. Med. Rep. 2016, 13, 2187-2193, doi:10.3892/mmr.2016.4796.

Publisher's Note: MDPI stays neutral with regard to jurisdictional claims in published maps and institutional affiliations.

(C) 2020 by the authors; licensee MDPI, Basel, Switzerland. This article is an open access article distributed under the terms and conditions of the Creative Commons by Attribution (CC-BY) license (http://creativecommons.org/licenses/by/4.0/). 